\title{
A NEW CONVERGENCE RATE FOR THE QUADRATURE METHOD FOR SOLVING SINGULAR INTEGRAL EQUATIONS
}

\author{
PETER JUNGHANNS \\ Technische Universität Karl-Marx-Stadt, Sektion Mathematik \\ PSF 964, DDR-Karl-Marx-Stadt 9010
}

\section{Introduction}

Let us consider a singular integral equation of the Cauchy type

$$
a(x) u(x)+\frac{b(x)}{\pi} \int_{-1}^{1} \frac{u(t)}{t-x} d t+\frac{1}{\pi} \int_{-1}^{1} h(x, t) u(t) d t=f(x),
$$

$-1 \leqslant x \leqslant 1$, where $a(x)$ and $b(x)$ are real-valued Hölder continuous coefficients with $a^{2}(x)+b^{2}(x)>0$ for $-1 \leqslant x \leqslant 1$. The functions $h(x, t)$ and $f(x)$ are the given kernel of the regular part of the integral operator defined by the left-hand side of (1) and the given right-hand side of (1), respectively. Define a continuous function

$$
g(x)=\frac{1}{2 \pi i} \ln \frac{a(x)-i b(x)}{a(x)+i b(x)}
$$

and a weight function

$$
\sigma(x)=\frac{(1-x)^{\lambda_{0}}(1+x)^{\mu_{0}}}{r(x)} \exp \int_{-1}^{1} \frac{g(t)}{t-x} d t,
$$

where $r(x)=\left(a^{2}(x)+b^{2}(x)\right)^{1 / 2}>0$ and $\lambda_{0}$ and $\mu_{0}$ are integers such that

$$
-1<\alpha:=\lambda_{0}+g(1), \quad \beta:=\mu_{0}-g(-1)<1 \text {. }
$$

Then the representation

$$
\sigma(x)=(1-x)^{\alpha}(1+x)^{\beta} w_{0}(x)
$$

is valid, where $w_{0}(x)$ is continuous and positive on $[-1,1]$. Furthermore, we make the following assumptions (cf. [1], [2]): 
$1^{\circ}$ There is a function

$$
c(x)=c_{0}(x) \prod_{j=0}^{N+1}\left|x-x_{j}\right|^{-\alpha_{j}},
$$

$-1=x_{N+1}<x_{N}<\therefore<x_{1}<x_{0}=1,-1 \leqslant x \leqslant 1$, such that

$$
B(x)=c(x) b(x), \quad-1 \leqslant x \leqslant 1,
$$

is a polynomial.

$2^{\circ}$ The exponents $\alpha_{j}(j=1, \ldots, N)$ are greater than -1 , and, furthermore, $\alpha_{0}-\alpha, \alpha_{N+1}-\beta, \alpha_{0}+\alpha, \alpha_{N+1}+\beta>-1$, and $c_{0}(x)>0$ is continuous on $[-1,1]$.

Now, for $-1<x<1$, equation (1) is equivalent to

(3) $a(x) \sigma(x) v(x)+\frac{B(x)}{\pi} \int_{-1}^{1} \frac{v(t)}{t-x} \sigma_{1}(t) d t+\frac{1}{\pi} \int_{-1}^{1} h_{1}(x, t) v(t) \sigma_{1}(t) d t=f_{1}(x)$,

where

$$
\begin{gathered}
u(t)=\sigma_{1}(t) v(t), \quad \sigma_{1}(t)=\sigma(t) / c(t), \\
h_{1}(x, t)=c(x) h(x, t), \quad f_{1}(x)=c(x) f(x) .
\end{gathered}
$$

If we define a linear bounded operator $A$ in the pair of Hilbert spaces $\left(L_{\sigma_{1}}^{2}, L_{\mu_{1}}^{2}\right)$, where

$$
\mu_{1}(x)=\mu(x) / c(x), \quad \mu(x)=1 / \sigma(x) r^{2}(x),
$$

by

$$
(A v)(x)=a(x) \sigma(x) v(x)+\frac{B(x)}{\pi} \int_{-1}^{1} \frac{v(t)}{t-x} \sigma_{1}(t) d t, \quad-1<x<1,
$$

then $A^{(-1)}$ given by

$$
\left(A^{(-1)} g\right)(t)=a(t) \mu(t) g(t)-\frac{B(t)}{\pi} \int_{-1}^{1} \frac{g(x)}{x-t} \mu_{1}(x) d x, \quad-1<t<1,
$$

is at least a one-sided inverse of $A$.

Lemma $1\left([7], \S 5,5^{\circ}-7^{\circ}\right)$. Assume that a function $\psi(x, t)$ is Hölder continuous with exponent $\gamma, 0<\gamma<1$, uniformly in the variables $x$ and t, i.e.

$$
\left|\psi\left(x^{\prime}, t^{\prime}\right)-\psi\left(x^{\prime \prime}, t^{\prime \prime}\right)\right| \leqslant C_{1}\left(\left|x^{\prime}-x^{\prime \prime}\right|^{\gamma}+\left|t^{\prime}-t^{\prime \prime}\right|^{\gamma}\right)
$$

(the $C_{k}$ denote positive constant numbers), $x^{\prime}, x^{\prime \prime} \in\left[a_{1}, b_{1}\right], t^{\prime}, t^{\prime \prime} \in\left[a_{2}, b_{2}\right]$, 
$-\infty<a_{j}, b_{j}<\infty(j=1,2)$. Let $x_{0} \in\left[a_{1}, b_{1}\right], 0<\delta<\gamma$, and

$$
\varphi(x, t)=\frac{\psi(x, t)-\psi\left(x_{0}, t\right)}{\left|x-x_{0}\right|^{\delta}} .
$$

Then there exists a constant $C_{2}$ such that

$$
\left|\varphi\left(x^{\prime}, t^{\prime}\right)-\varphi\left(x^{\prime \prime}, t^{\prime \prime}\right)\right| \leqslant C_{2}\left(\left|x^{\prime}-x^{\prime \prime}\right|^{\gamma-\delta}+\left|t^{\prime}-t^{\prime \prime}\right|^{\gamma-\delta}\right)
$$

for $x^{\prime}, x^{\prime \prime}, t^{\prime}, t^{\prime \prime}$ as above.

Corollary 1. Assume that $\psi(x, t)$ possesses the same property as in Lemma $1\left(a_{j}=-1, b_{j}=1\right), \quad 0<\delta_{k}<\gamma, \psi\left(x_{k}, t\right)=0(k=0,1, \ldots, N+1$; $t \in[-1,1])$, and

$$
\Phi(x, t)=\frac{\psi(x, t)}{\prod_{k=0}^{N+1}\left|x-x_{k}\right|^{\delta_{k}}}
$$

Then

$$
\left|\Phi\left(x^{\prime}, t^{\prime}\right)-\Phi\left(x^{\prime \prime}, t^{\prime \prime}\right)\right| \leqslant C_{31}\left(\left|x^{\prime}-x^{\prime \prime}\right|^{\gamma-\delta}+\left|t^{\prime}-t^{\prime \prime}\right|^{\gamma-\delta}\right),
$$

$x^{\prime}, x^{\prime \prime}, t^{\prime}, t^{\prime \prime} \in[-1,1]$, where $\delta=\max _{0 \leqslant k \leqslant N+1} \delta_{k}$.

Proof. We apply Lemma 1 to the intervals

$$
\left[-1, \frac{1}{2}\left(x_{N}+x_{N-1}\right)\right],\left[\frac{1}{2}\left(x_{N}+x_{N-1}\right), \frac{1}{2}\left(x_{N-1}+x_{N-2}\right)\right], \ldots,\left[\frac{1}{2}\left(x_{2}+x_{1}\right), 1\right] \text {. }
$$

LEMMA 2. If $\left|f\left(x^{\prime}\right)-f\left(x^{\prime \prime}\right)\right| \leqslant C_{4}\left|x^{\prime}-x^{\prime \prime}\right|^{\gamma}, x^{\prime}, x^{\prime \prime} \in\left[a_{1}, b_{1}\right], \quad 0<\gamma<1$, $\delta>0$, and $x_{0} \in\left[a_{1}, b_{1}\right], f\left(x_{0}\right)=0$, then there is a constant $C_{5}$ such that

$$
\left|g\left(x^{\prime}\right)-g\left(x^{\prime \prime}\right)\right| \leqslant C_{5}\left|x^{\prime}-x^{\prime \prime}\right|^{\prime}, \quad x^{\prime}, x^{\prime \prime} \in\left[a_{1}, b_{1}\right],
$$

where $g(x)=f(x)\left|x-x_{0}\right|^{\delta}$.

Proof. In the case $\delta \geqslant \gamma$ the validity of the assertion is clear. Now, assume $\delta<\gamma$. Let $x_{0} \leqslant x^{\prime}<x^{\prime \prime}$. If $x_{0}=x^{\prime}$, then

$$
\begin{aligned}
\left|g\left(x^{\prime}\right)-g\left(x^{\prime \prime}\right)\right| & =\left|f\left(x^{\prime \prime}\right)\left(x^{\prime \prime}-x_{0}\right)^{\delta}\right| \leqslant C_{4}\left(x^{\prime \prime}-x_{0}\right)^{\gamma+\delta} \\
& \leqslant C_{51}\left(x^{\prime \prime}-x^{\prime}\right)^{\gamma} .
\end{aligned}
$$

In the other case $\left(x_{0}<x^{\prime}<x^{\prime \prime}\right)$ it follows that

$$
\begin{aligned}
\left|g\left(x^{\prime}\right)-g\left(x^{\prime \prime}\right)\right| & =\left|\left(x^{\prime}-x_{0}\right)^{\delta} f\left(x^{\prime}\right)-\left(x^{\prime \prime}-x_{0}\right)^{\delta} f\left(x^{\prime \prime}\right)\right| \\
& =\left(\left(x^{\prime \prime}-x_{0}\right)^{\delta}-\left(x^{\prime}-x_{0}\right)^{\delta}\right)\left|f\left(x^{\prime}\right)\right|+\left(x^{\prime \prime}-x_{0}\right)^{\delta}\left|f\left(x^{\prime}\right)-f\left(x^{\prime \prime}\right)\right| .
\end{aligned}
$$


First, let $x^{\prime}-x_{0} \leqslant x^{\prime \prime}-x^{\prime}$. Thus,

$$
\begin{aligned}
\left|g\left(x^{\prime}\right)-g\left(x^{\prime \prime}\right)\right| & \leqslant C_{52}\left|f\left(x^{\prime}\right)\right|+C_{53}\left|f\left(x^{\prime}\right)-f\left(x^{\prime \prime}\right)\right| \\
& \leqslant C_{52} C_{4}\left(x^{\prime}-x_{0}\right)^{\gamma}+C_{53} C_{4}\left(x^{\prime \prime}-x^{\prime}\right)^{\gamma} \\
& \leqslant C_{54}\left(x^{\prime \prime}-x^{\prime}\right)^{\gamma}
\end{aligned}
$$

Secondly, if $x^{\prime \prime}-x^{\prime}<x^{\prime}-x_{0}$, then there is an $\tilde{x}, x^{\prime}<\tilde{x}<x^{\prime \prime}$, with

$$
\begin{aligned}
\left|g\left(x^{\prime}\right)-g\left(x^{\prime \prime}\right)\right| & \leqslant \delta\left(\tilde{x}-x_{0}\right)^{\delta-1}\left(x^{\prime \prime}-x^{\prime}\right) \cdot C_{4}\left(x^{\prime}-x_{0}\right)^{\gamma}+C_{53} C_{4}\left(x^{\prime \prime}-x^{\prime}\right)^{\gamma} \\
& \leqslant\left(C_{4} \delta\left(x^{\prime}-x_{0}\right)^{\delta}+C_{53} C_{4}\right)\left(x^{\prime \prime}-x^{\prime}\right)^{\gamma} \leqslant C_{55}\left(x^{\prime \prime}-x^{\prime}\right)^{\gamma} .
\end{aligned}
$$

In the case $x^{\prime}<x^{\prime \prime} \leqslant x_{0}$, we proceed analogously, and (4) follows.

Corollary 1 and Lemma 2 imply

Corollary 2. Let $\psi(x, t)$ satisfy the assumptions of Lemma 1. Furthermore, assume $\delta_{k}<\gamma, \psi\left(x_{k}, t\right)=0(k=0,1, \ldots, N+1 ; t \in[-1,1])$, and

$$
\Phi(x, t)=\frac{\psi(x, t)}{\prod_{k=0}^{N+1}\left|x-x_{k}\right|^{\delta_{k}}}
$$

Then

$$
\left|\Phi\left(x^{\prime}, t^{\prime}\right)-\Phi\left(x^{\prime \prime}, t^{\prime \prime}\right)\right| \leqslant C_{32}\left(\left|x^{\prime}-x^{\prime \prime}\right|^{\gamma-\delta}+\left|t^{\prime}-t^{\prime \prime}\right|^{\gamma-\delta}\right),
$$

$x^{\prime}, x^{\prime \prime}, t^{\prime}, t^{\prime \prime} \in[-1,1]$, where $\delta=\max \left\{0, \delta_{0}, \ldots, \delta_{N+1}\right\}$.

Proof. Analogous to the proof of Corollary 1.

Lemma $3\left([7], \S 18,3^{\circ}\right)$. Let $\psi(x, t)$ fulfil the assumptions of Lemma 1 with $a_{1}=a_{2}=-1, b_{1}=b_{2}=1$. Then the function

$$
G(t)=\int_{-1}^{1} \frac{\psi(x, t)}{x-t} d x
$$

is Hölder continuous with exponent $\gamma$ on $[-1,1]$ if $\psi(-1, t)=\psi(1, t)=0$ $(i \in[-1,1])$.

The fact that a function $f(t),-1 \leqslant t \leqslant 1$, has a derivative of order $m$ ( $m$ a nonnegative integer), Hölder continuous with exponent $\gamma, 0<\gamma<1$, will be denoted by $f \in C^{m, \gamma}$.

Lemma $4\left([2], \S 3 ;[5]\right.$, p. 91). For each polynomial $p(x),\left(A^{(-1)} p\right)(t)$ is again a polynomial. 


\section{A regularity property of the solution of}

a singular integral equation

Lemma 5. If $a, b \in C^{0, \gamma}$, then $w_{0} \in C^{0, \gamma}$ (cf. (2)).

Proof. Note that $r, g \in C^{0, y}$ and write

$$
\begin{aligned}
\int_{-1}^{1} \frac{g(t)}{t-x} d t= & \int_{-1}^{1} \frac{g(t)-((1+t) g(1)+(1-t) g(-1)) / 2}{t-x} d t \\
& +\frac{1}{2} \int_{-1}^{1}\left\{\frac{(1+x) g(1)+(1-x) g(-1)}{t-x}+g(1)-g(-1)\right\} d t .
\end{aligned}
$$

Denote the first integral on the right-hand side by $\tilde{g}(x)$. Then $\tilde{g} \in C^{0 . \gamma}$ in view of Lemma 3. It follows that

$$
\begin{aligned}
\sigma(x)=\frac{(1-x)^{\lambda_{0}}(1+x)^{\mu_{0}}}{r(x)}\left(\frac{1-x}{1+x}\right)^{(1+x) g(1) / 2+(1-x) g(-1) / 2} & \\
& \times \exp (\tilde{g}(x)+g(1)-g(-1))
\end{aligned}
$$

or

$$
\begin{aligned}
w_{0}(x)=(1-x)^{(1-x)(g(-1)-g(1)) / 2}(1+x)^{(1+x)(\dot{g}(1)-g(-1)) / 2} & \\
& \times \frac{\exp (\tilde{g}(x)+g(1)-g(-1))}{r(x)} .
\end{aligned}
$$

Because $e^{s \ln s} \in C^{0 . \varepsilon}$ for $s \in[0,1]$ and all $\varepsilon \in(0,1)$ the assertion follows immediately.

ThEOREM 1. Let $a, b, c_{0} \in C^{0, \eta}$, and

$$
\max \left\{0, \alpha-\alpha_{0}, \beta-\alpha_{N+1},-\alpha_{1}, \ldots,-\alpha_{N}\right\}<\gamma<1 .
$$

Assume $f \in C^{m, \gamma}$. Then

$$
A^{(-1)} f \in C^{m, \delta}
$$

where $\delta=\min \left\{\eta, \gamma, \gamma+\alpha_{0}-\alpha, \gamma+\alpha_{N+1}-\beta, \gamma+\alpha_{1}, \ldots, \gamma+\alpha_{N}\right\}$.

Proof. On taking into account the equality

$$
\begin{aligned}
\left(A^{(-1)} f\right)(t)= & f(t)\left[a(t) \mu(t)-\frac{B(t)}{\pi} \int_{-1}^{1} \frac{\mu_{1}(x)}{x-t} d x\right] \\
& -\frac{B(t)}{\pi} \int_{-1}^{1} \frac{f(x)-f(t)}{x-t} \mu_{1}(x) d x
\end{aligned}
$$


and Lemma 4 it remains to prove that

$$
B F \in C^{m, \delta} \text {, }
$$

where

$$
F(t)=\frac{1}{\pi} \int_{-1}^{1} \frac{f(x)-f(t)}{x-t} \mu_{1}(x) d x .
$$

By induction it can be proved that

$$
\frac{d^{k}}{d t^{k}}\left[\frac{f(x)-f(t)}{x-t}\right]=\frac{k !\left[f(x)-\sum_{j=0}^{k} \frac{f^{(t)}(t)}{j !}(x-t)^{j}\right]}{(x-t)^{k+1}}
$$

$k=0,1, \ldots, m$. Thus,

$$
F^{(k)}(t)=\frac{k !}{\pi} \int_{-1}^{1} \frac{f(x)-\sum_{j=0}^{k} \frac{f^{(j)}(t)}{j !}(x-t)^{j}}{(x-t)^{k+1}} \mu_{1}(x) d x,
$$

and (4) is valid if

$$
B F^{(m)} \in C^{0, \delta} \text {. }
$$

Setting

$$
\Phi(x, t)=m !\left[f(x)-\sum_{j=0}^{m-1} \frac{f^{(j)}(t)}{j !}(x-t)^{j}\right] /(x-t)^{m}
$$

we obtain

$$
\begin{aligned}
\Phi(x, t) & =\frac{m}{(x-t)^{m}} \int_{t}^{x} f^{(m)}(y)(x-y)^{m-1} d y \\
& =m \int_{0}^{1} f^{(m)}(s t+(1-s) x) s^{m-1} d s .
\end{aligned}
$$

Using the inequality

$$
\left(r_{1}+r_{2}\right)^{\gamma} \leqslant 2^{1-\gamma}\left(r_{1}^{\gamma}+r_{2}^{\gamma}\right), \quad r_{1} \geqslant 0, r_{2} \geqslant 0
$$

([7], §5), we conclude that

$$
\left|\Phi\left(x^{\prime}, t^{\prime}\right)-\Phi\left(x^{\prime \prime}, t^{\prime}\right)\right| \leqslant C_{6}\left(\left|x^{\prime}-x^{\prime \prime}\right|^{\gamma}+\left|t^{\prime}-t^{\prime \prime}\right|^{\gamma}\right),
$$

$x^{\prime}, x^{\prime \prime}, t^{\prime}, t^{\prime \prime} \in[-1,1]$ (cf. [3], p. 138). Let

$$
\Psi(x, t)=\Phi(x, t)-f^{(m)}(t) .
$$

We define

$$
P(x, t)=\sum_{j=0}^{N+1} \Psi\left(x_{j}, t\right) \prod_{\substack{k=0 \\ k \neq j}}^{N+1} \frac{x-x_{k}}{x_{j}-x_{k}}
$$


which is a polynomial with respect to the variable $x$. Now, we can write $B F^{(m)}$ as follows:

$$
\begin{aligned}
B(t) F^{(m)}(t)= & \frac{B(t)}{\pi} \int_{-1}^{1} \frac{(\Psi(x, t)-P(x, t)) \mu_{1}(x)}{x-t} d x \\
& -\left[a(t) \mu(t) P(t, t)-\frac{B(t)}{\pi} \int_{-1}^{1} \frac{P(x, t)}{x-t} \mu_{1}(x) d x\right] \\
& +a(t) \mu(t) P(t, t) .
\end{aligned}
$$

In view of Lemma 5 the representation

$$
\mu_{1}(x)=(1-x)^{\alpha_{0}-\alpha}(1-x)^{\alpha_{N}-1-\beta} \prod_{j=1}^{N}\left|x-x_{j}\right|^{\alpha_{j}} c_{0}(x) /\left(w_{0}(x) r^{2}(x)\right)
$$

holds with $c_{0} /\left(w_{0} r^{2}\right) \in C^{0, \eta}$. According to Corollary 2 and Lemma 3,

$$
\int_{-1}^{1} \frac{(\Psi(x, t)-P(x, t)) \mu_{1}(x)}{x-t} d x \in C^{0, \delta}
$$

Furthermore, by Lemma 4 the term in square brackets in (7) also belongs to $C^{0, \delta}$. Finally, from (6) we obtain $\Phi(t, t)=f^{(m)}(t)$, from which we conclude that $P(-1,-1)=\Psi(-1,-1)=0, P(1,1)=\Psi(1,1)=0$, and (by Corollary 2)

$$
a(t) \mu(t) P(t, t) \in C^{0, \delta}
$$

This proves (5).

\section{The Gauss quadrature method and rates of convergence}

By definition, $x=-\left(\lambda_{0}+\mu_{0}\right)$ is the index of equation (3). In case $x>0$ we require that $v(t)$ fulfils the additional conditions

$$
\frac{1}{\pi} \int_{-1}^{1} v(t) t^{l} \sigma_{1}(t) d t=0 \quad(l=0,1, \ldots, x-1) .
$$

In what follows we prove that (3) (with (8) in case $x>0$ ) is uniquely solvable in $L_{\sigma_{1}}^{2}$. Denote by $t_{k}(k=1, \ldots, n)$ and $x_{j}(j=1, \ldots, n-x)$ the zeros of the orthogonal polynomials of degree $n$ and $n-x$ with respect to the weights $\sigma_{1}(t)$ and $\mu_{1}(x)$, respectively. We seek an approximate solution of (3) in the form

$$
v_{n}(t)=\sum_{k=1}^{n} v_{n}\left(t_{k}\right) \prod_{\substack{i=1 \\ i \neq k}}^{n} \frac{t-t_{i}}{t_{k}-t_{i}}
$$


by solving the algebraic equations

$$
\sum_{k=1}^{n} \lambda_{k}\left[\frac{B\left(x_{j}\right)}{t_{k}-x_{j}}+h\left(x_{j}, t_{k}\right)\right] v_{n}\left(t_{k}\right)=f_{1}\left(x_{j}\right) \quad(j=1, \ldots, n-x),
$$

where the weights $\lambda_{k}$ are given via the Gauss quadrature rule

$$
\frac{1}{\pi} \int_{-1}^{1} v(t) \sigma_{1}(t) d t \sim \sum_{k=1}^{n} \lambda_{k} v\left(t_{k}\right)
$$

In case $x>0$ we complete (9), for example, by

$$
\sum_{k=1}^{n} \lambda_{k} v_{n}\left(t_{k}\right) t_{k}^{l}=0(l=0,1, \ldots, x-1) \text {. }
$$

If $x<0$ we modify (9) so that the vector

$$
\left(v_{n}\left(t_{1}\right), \ldots, v_{n}\left(t_{n}\right), \xi_{1}, \ldots, \xi_{-x}\right)^{T}
$$

is to be determined by solving

$$
\sum_{k=1}^{n} \lambda_{k}\left|\frac{B\left(x_{j}\right)}{t_{k}-x_{j}}+h\left(x_{j}, t_{k}\right)\right| v_{n}\left(t_{k}\right)+\sum_{l=1}^{-x} a_{j l} \check{\zeta}_{l}=f_{1}\left(x_{j}\right)
$$

where the columns $\left(a_{1 l}, \ldots, a_{n-x, l}\right)^{T}$. are chosen in such a way that

$$
\sum_{j=1}^{n-x} \gamma_{j} \lambda_{k}\left|\frac{B\left(x_{j}\right)}{t_{k}-x_{j}}+h\left(x_{j}, t_{k}\right)\right| a_{j l}=0 \quad(k=1, \ldots, n ; l=1, \ldots,-x),
$$

and

$$
\sum_{j=1}^{n-x} \gamma_{j} a_{j l} a_{j m}=\delta_{l m} \quad(l, m=1, \ldots,-x) .
$$

THEOREM 2 ([3], Theorem 3.1). Assume $c(x) \equiv 1$ (i.e. $b(x)$ is a polynomial), $\alpha, \beta<0, \sigma=\sigma_{1} \in C^{0, \varepsilon_{1}}$, and also $h(x, \cdot), h(\cdot, t) \in C_{?}^{m, \gamma}$ (uniformly with respect to the variables $x$ and $t$, respectively). Then for sufficiently large $n$ the equations (9) (with (11) in case $x>0$ ) or $\left(9^{\prime}\right)$ are uniquely solvable, and

$$
\max _{1 \leqslant k \leqslant n}\left|v\left(t_{k}\right)-v_{n}\left(t_{k}\right)\right| \leqslant C_{7} n^{-(m+\varepsilon)} \ln n,
$$

where $\varepsilon=\min \left(\gamma, \varepsilon_{1}\right)$.

Using Theorem 1 and the same arguments as in [3] one can prove

THEOREM 3. Let $f_{1}$ and $h_{1}$ fulfil the same conditions as $f$ and $h$ in Theorem 2. Furthermore, assume $a, b, c_{0} \in C^{0, \eta}$ and $\alpha_{0}-\alpha, \alpha_{N-1}-\beta, \alpha_{2}, \ldots, \alpha_{N} \geqslant 0$. Then for sufficiently large $n$ the equations (9) or (9) with (11) or (9') are

(1) $\gamma_{j}$ are the Christoffel numbers with respect to $\mu_{1}(x)$. 
uniquely solvable, and

$$
\max _{1 \leqslant k \leqslant n}\left|v\left(t_{k}\right)-v_{n}\left(t_{k}\right)\right| \leqslant C_{8} n^{-(m+\delta)} \ln n,
$$

where $\delta=\min (\gamma, \eta)$.

Let us consider the case of constant real coefficients $a$ and $b$ with $a^{2}+b^{2}=1$ and $b>0$. Then, by applying Theorems 2 and 3 it is only possible to investigate the index case $x=1$, where

$$
\sigma(x)=(1-x)^{-\varepsilon^{\prime}}(1-x)^{\varepsilon^{\prime}-1},
$$

$a+i b=e^{i \pi \varepsilon^{\prime}}, \quad 0<\varepsilon^{\prime}<1$. From Theorem 2 we obtain (12) with $\varepsilon$ $=\min \left(\gamma, \varepsilon^{\prime}, 1-\varepsilon^{\prime}\right)$, while Theorem 3 gives (13) with $\delta=\gamma$.

Remark. With the help of Theorem 3 the estimate of the global error

$$
\max _{-1 \leqslant t \leqslant 1}\left|v(t)-v_{n}(t)\right|
$$

can also be improved using the method of [3], $\$ 4$.

\section{References}

[1] D. Elliott, The classical collocation method for singular integral equations, SIAM J. Numer. Anal. 19 (1982), 816-832.

[2] -, Orthogonal polynomials associated with singular integral equations having a Cauchy kernel, SIAM J. Math. Anal. 13 (1982), 1041-1052.

[3] -, Rates of convergence for the method of classical collocation for solving singular integral equations, SIAM J. Numer. Anal. 21 (1984), 136-148.

[4] P. Junghanns, Uniform convergence of approximate methods for Cauchy-type singular - integral equations over $(-1,1)$. Wiss. Zeitschr. TH Karl-Marx-Stadt, 26 (1984), 251-256.

[5] -, Some remarks on the zero distribution of pairs pf polynomials associated with singular integral operators. A convergence theorem for the quadrature method, ibid. 27 (1985), 88-93.

[6] P. Junghanns and B. Silbermann, Zur Theorie der Näherungsverfahren für singuläre Integralgleichungen auf Intervallen, Math. Nachr. 103 (1981), 199-244.

[7] N. I. Muschelischwili, Singuläre Integralgleichungen, Berlin 1965.

Presented to the Semester Approximation and Function Spaces

February 27-May 27, 1986 